EDITORIAL

\title{
Status of maternal health in Nepal
}

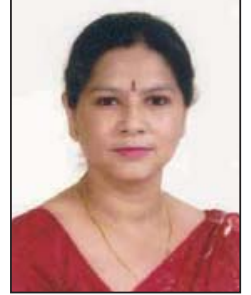

Achala Vaidya

Maternity Hospital, Thapathali
Every minute of every day, somewhere in the world, a woman dies as a result of complications arising during pregnancy and child birth. For women, human rights include access to services that will ensure safe pregnancy and childbirth.

Since the 1940's, maternal deaths have become increasingly rare in developed countries. The same cannot be said, however, of developing areas, where the persistence of high levels of maternal mortality is symptomatic of a pervasive neglect of women's most fundamental human rights. Such neglect affects most acutely the poor, the disadvantaged and the powerless. For more than half a million of women, death is the last episode in a long story of pain and suffering; million more women are disabled and damaged, many of them for rest of their lives. The suffering often goes beyond the purely physical and affects women's ability to undertake their social and economic responsibilities and to share in the development of their communities.

Maternal death is a tragedy for individual woman, for families and for their communities.

Women are the main stays of families, the key educators of children, health care providers, carers of young and old alike, farmers, traders, and often the main, if not the sole, bread earners. A society deprived of the contribution made by women is one that will see its social and economic life decline, its culture impoverished, and its potential for development severely limited.

In 1987, The First International Safe Motherhood Conference took place in Nairobi and the goal of a $50 \%$ reduction in 1990 levels of maternal mortality by the year 2000 was formulated. This goal was later adopted by national governments and by other international conferences, including the World Summit for Children in New York in 1990, the International Conference on Population and Development in Cairo in 1994, and the
Fourth World Conference on Women in Beijing in 1995. Much more is known now than it was 10 years ago about the interventions that are effective, the barriers to access care, the constraints on implementation of programmes, and the specific elements of care that must be provided. The lessons that have been learned were highlighted at an international Technical Consultation held in Colombo, Sri Lanka, in October 1997 to mark the tenth anniversary of the Safe Motherhood Initiative. In the course of Consultation, the United Nations agencies most closely involved in the development and implementation of reproductive health programmes reached consensus on the measures that work, what they cost, and how they can be effectively implemented. This joint statement reflects that consensus and presents the way forward for everyone concerned with any aspect of safe motherhood.

The death of a woman during pregnancy or childbirth is not only a health issue but also a matter of social injustice.

A maternal death is the death of a woman while pregnant or within 42 days of termination of pregnancy, regardless of the site or duration of pregnancy, from any cause related to or aggravated by the pregnancy or its management. Maternal deaths are subdivided into direct and indirect obstetric deaths. Direct obstetrics deaths results from obstetrics complications of pregnancy, labour, or the postpartum period. They are usually due to one of the five major causes haemorrhage (usually occurring postpartum), sepsis, eclampsia, obstructed labour, and complications of unsafe abortion- as well as interventions, omissions, incorrect treatment, or events resulting from any of these. Indirect obstetrics deaths result from previously existing diseases or from diseases arising during pregnancy (but without direct obstetrics causes), which were aggravated by the physiological effects of pregnancy; examples of such diseases include malaria, anaemia, HIV/AIDS, and cardio vascular disease. 
Maternal mortality ratio represents the risk associated with each pregnancy, i.e. the obstetric risk. It is calculated as the number of maternal deaths during a given year per 100000 live births during the same period. Although the measure had traditionally been referred to as a rate it is actually a ratio and is now usually called such by researchers.

Maternal mortality rate measures both the obstetric risk and the frequent with which women are exposed to this risk. It is calculated as the number of maternal deaths in a given period per 100000 women of reproductive age (usually 15-49 years).

The terms "ratio" and "rate" are often used interchangeably: for the sake of clarity it is therefore essential referring to either of these measures of maternal mortality, to specify the denominator used.

Lifetime risk of maternal death takes into account both the probability of dying as a result of the pregnancy cumulated across a woman's reproductive years.

The first estimates of the extent of maternal mortality around the world were made around the 1980's. They indicated that globally some 500000 die each year from pregnancy related causes. In 1996, WHO and UNICEF revised the estimates for 1990 on the basis of the growing volume of information that has become available in recent years. These new estimates showed that the scale of the problem was significantly greater than had originally been suspected and that closer to 600000 maternal deaths occur each year, with the overwhelming majority of them in developing countries. In developed countries, the maternal mortality ratio averages around 27 maternal deaths per 100000 live births; in developing countries the ratio is nearly 20 times higher, at 480 per 100000 , and maybe as high as 1000 per 100000 in some regions.

The medical causes of maternal deaths are similar throughout the world.

Globally, around $80 \%$ of all maternal deaths are the direct results of complications arising during pregnancy, delivery, or the puerperium. The single most common cause accounting for a quarter of all maternal deathsis severe bleeding, generally occurring postpartum especially postpartum haemorrhage, is unpredictable, sudden in onset, and more dangerous when a woman is a anaemic. Globally, some $25 \%$ of all maternal deaths are due to haemorrhage. Blood loss can very rapidly lead to death in absence of prompt and appropriate life saving care which includes the administration of drugs to control bleeding, massage of the uterus to stimulate contractions, and blood transfusion if necessary.

Sepsis, which is often a consequence of poor hygiene during delivery or of untreated sexually transmitted diseases (STDs), accounts for some $15 \%$ of maternal deaths. Such infections can be effectively prevented by careful attention to clean delivery and by detection and management of STDs during pregnancy. Systemic postpartum care will ensure rapid detection of infection and its management by appropriate antibiotics.

Hypertensive disorders of pregnancy, particularly eclampsia (convulsions), are the causes of approximately $12 \%$ of all maternal deaths? Deaths from hypertensive disorders can be prevented by careful monitoring during pregnancy and by treatment with relatively simple anticonvulsant drugs (e.g. Magnesium sulphate) in cases of eclampsia.

Prolonged or obstructed labour accounts for about $8 \%$ of maternal deaths. This is often caused by cephalopelvic disproportion or by abnormal lie. Disproportion is more common where malnutrition is endemic, especially among populations with various traditions and taboos regarding the diet of girls and women. It is worse where girls marry young and are expected to prove their fertility, often before they are fully grown.

Complications of unsafe abortions are responsible for a substantial proportion (13\%) of maternal deaths. In some parts of the world, one-third or more of all maternal deaths are associated with unsafe abortions. These deaths can be prevented if women have access to family planning information and services, care for abortion related complications, and, where abortion is not prohibited by law, safe abortion care.

Approximately $20 \%$ of maternal deaths are the results of pre-existing conditions that are exacerbated by pregnancy or its management. One of the most significant of these indirect causes of death is anaemia which, as well as causing death through cardiovascular arrest, is thought also to underlie a substantial proportion of direct deaths (particularly those due to haemorrhage and sepsis). Other indirect causes of death include malaria, hepatitis, heart diseases, and, increasingly in some settings, HIV/AIDS. Many of these conditions are relative or absolute contraindications for pregnancy.

Women need to be informed of these problems and enabled to prevent further pregnancies while the conditions are being treated. 
The maternal mortality ratio (MMR) of Nepal is among the highest in the world. The 1996 Nepal Family Health Survey revealed a MMR of 539 per 100,000 live births, which is still considered to be the most reliable estimate so far. In Nepal, approximately $80-90 \%$ of births take place at home, often "conducted" by family members, sometimes assisted by a traditional birth attendant (TBA), but many without any attendant at all. The proportion of all births assisted by a trained health worker, but not necessarily one who has the full range of skills to be counted as a skill birth attendant, both at home and in an institution has increased, from $8 \%$ in $2001 / 02$ to $18 \%$ in2003/04. Currently $9.6 \%$ of births take place in an institution. According to the 1998 Maternal Mortality and Morbidity Study, conducted in 3 districts of the country, where safe motherhood activities were underway ( Kailali, Rupandehi, Okhaldhunga), the majority of women, who died as a result of pregnancy, childbirth or the postpartum period, did so at home (67.4\%). Slightly more then $11 \%$ of deaths occurred on the way to the health facility and about $21 \%$ in a health facility (14.4\% in a hospital, $4.5 \%$ in a private clinic and $2.3 \%$ in a primary health centre (PHC)
The majority of maternal deaths $(62.1 \%)$ take place soon after birth. According to the same study as above, postpartum haemorrhage was the number one cause of direct maternal death $(46.3 \%)$, followed by obstructed labour (16.3\%), eclampsia (14.3\%) and puerperal sepsis $(11.8 \%)$.Abortion was the lading cause of hospital admission, followed by postpartum haemorrhage.

Nepal's Safe Motherhood Programme is coordinated by the Family Health Division of the Directorate of Health Services of $\mathrm{MOH}$, within the context of the National Reproductive Health Programme. Under this programme a Safe Motherhood Committee has been established, in which most stakeholders participate and contribute, amongst others, to policy and strategy development. The strategy that was adopted to reduce maternal mortality focuses on increased access to family planning, essential obstetric care, essential neonatal care, comprehensive safe abortion services and skilled birth attendants (SBAs). According to the Second Long-term Health Plan 1997-2017, the country intends to reduce the MMR to 250 by 2017 . 